\title{
An asymptotic analysis of chain-branching ignition in the laminar wake of a splitter plate separating streams of hydrogen and oxygen
}

\author{
A L Sánchez I Iglesias A Liñán:
}

\begin{abstract}
The chain-branching process leading to ignition in the high-temperature laminar wake that forms at the trailing edge of a thin splitter plate separating a stream of hydrogen from a stream of oxygen is investigated with a reduced chemistry description that employs $\mathbf{H}$ as the only chain-branching radical not in steady state. The analysis presented covers ignition events occurring in the Rott-Hakkinen and Goldstein regions, where self-similar solutions for the different flow variables are available. It is found that the initiation reactions, which create the first radicals, are only important in a relatively small initial region, becoming negligible downstream as the radical mole fractions increase to values larger than the ratio of the characteristic branching time to the characteristic initiation time, a very small quantity at temperatures of practical interest. As a result, most of the ignition history is controlled by the autocatalytic branching reactions, giving rise to a radical pool that increases exponentially with distance in a process that is described by using as a large parameter the ratio of the streamwise distance to the downstream extent of the initial region where initiation reactions are significant. Comparisons of the asymptotic results with numerical integrations of the conservation equations reveal that a three-term expansion for the $\mathrm{H}$-atom profile is necessary in this case to provide an accurate prediction for the ignition distance.
\end{abstract}

\section{Introduction}

The problem of autoignition of two unpremixed streams of oxidizer and fuel initially separated by a splitter plate has been widely studied in recent years. The character of the solution that emerges depends on the underlying chemistry, leading to either thermal explosions [1] or chain-branching explosions [2-5]. The former processes were first investigated by Liñán and Crespo [1], who adopted for the chemistry description a one-step Arrhenius model with large activation energy. Their analysis revealed the existence of an initial quasifrozen region in which the reactants mix and begin to react, followed by a sudden thermal runaway that clearly identifies the ignition location. A similar thermal-runaway behaviour was encountered by Sánchez [6], who extended the work of Liñan and Crespo [1] to take into account the wake associated with the finite boundary layers of the merging streams. In general, when two parallel streams merge at the end of a separating plate, there exists a momentum-deficit region in the 
vicinity of the trailing edge, of length comparable to that of the plate, where a longer residence time is available for chemical reactions to occur. The importance of the wake effect on the overall ignition process depends on the characteristic chemical time corresponding to the plate temperature and characteristic residence time associated with the wake [7].

Analytical studies of chain-branching explosions in the coflow mixing layer $[4,5]$ have been conducted in recent years for hydrogen-air systems with free-stream temperatures above the crossover temperature [8] at which the rate of the main chain-terminating reaction $\mathrm{H}+\mathrm{O}_{2}+\mathrm{M} \rightarrow \mathrm{HO}_{2}+\mathrm{M}$ equals that of the rate-controlling branching reaction $\mathrm{H}+\mathrm{O}_{2} \rightarrow$ $\mathrm{OH}+\mathrm{O}$. It was seen that, although initiation reactions, such as $\mathrm{H}_{2}+\mathrm{O}_{2} \rightarrow \mathrm{OH}+\mathrm{OH}$, are very slow, with characteristic times $t_{\mathrm{I}}$ much larger than those of branching $t_{\mathrm{B}}$ and recombination $t_{\mathrm{R}}$, they must be necessarily taken into account to guarantee the initial radical build up. For temperatures sufficiently above crossover, the rate of the three-body recombination reactions, responsible for most of the heat released in hydrogen-oxygen combustion, becomes much smaller than that of the branching reactions, thereby causing the mixing layer to be initially thermally frozen. Because of the relative scaling $t_{\mathrm{I}} \gg t_{\mathrm{R}} \gg t_{\mathrm{B}}$ of the relevant chemical times at these temperatures, the resulting igniting mixing layer exhibits a distinct structure $[4,5]$. There is a short chain-initiation region of characteristic length $U t_{\mathrm{B}}$, where $U$ is the characteristic free-stream velocity, in which radical concentrations are very small, and the slow initiation reactions control the process, followed by a long autocatalytic chain-branching region in which chain-carrier concentrations increase exponentially with downstream distance. This region of autocatalytic growth ends at the ignition point, located at a distance from the splitter plate of order $U t_{i}$, where $t_{i}=t_{\mathrm{B}} \ln \left(t_{1} / t_{\mathrm{B}}\right)$ is the characteristic ignition time. At the ignition point the mole fractions of the radicals achieve their peak values of order unity, corresponding to partial equilibrium of the branching reactions. Significant exothermicity occurs only downstream in a long region of characteristic length $U t_{\mathrm{R}}$ where radical recombination leads to the formation of a diffusion flame, a process analysed elsewhere [9].

The objective of this paper is to extend our previous analyses [4,5] by accounting for the effect of the wake that exists downstream of the splitter plate separating the two coflowing streams, with attention restricted to ignition events with ignition points located within the wake in two zones of self-similar flow, in the Goldstein region [10] and in the so-called Rott-Hakkinen region [11, 12]. A brief description of the trailing-edge flow-field structure and its associated characteristic scales is given in the following section. The governing equations are presented in section 3, followed by the asymptotic analysis in section 4. Comparisons of the asymptotic predictions with results of numerical integrations of the conservation equations are given in the next section. Finally, conclusions are given in section 6 .

\section{The flow field at the trailing edge of a splitter plate}

We consider the flow field that emerges at the trailing edge of a splitter plate of negligible thickness that separates a stream of oxygen and a stream of hydrogen, with dilution with an inert permitted in both streams. Because of the no-slip condition on the splitter-plate surface, on both sides of the plate there exist boundary layers of characteristic thickness $l_{B}$, across which the flow velocity evolves from a zero value at the plate to the freestream value as the transverse distance from the plate $y$ becomes large compared to $l_{\mathrm{B}}$. At the trailing edge the restraining effect of the plate is removed, causing the flow to accelerate as the two streams begin to mix and react. 


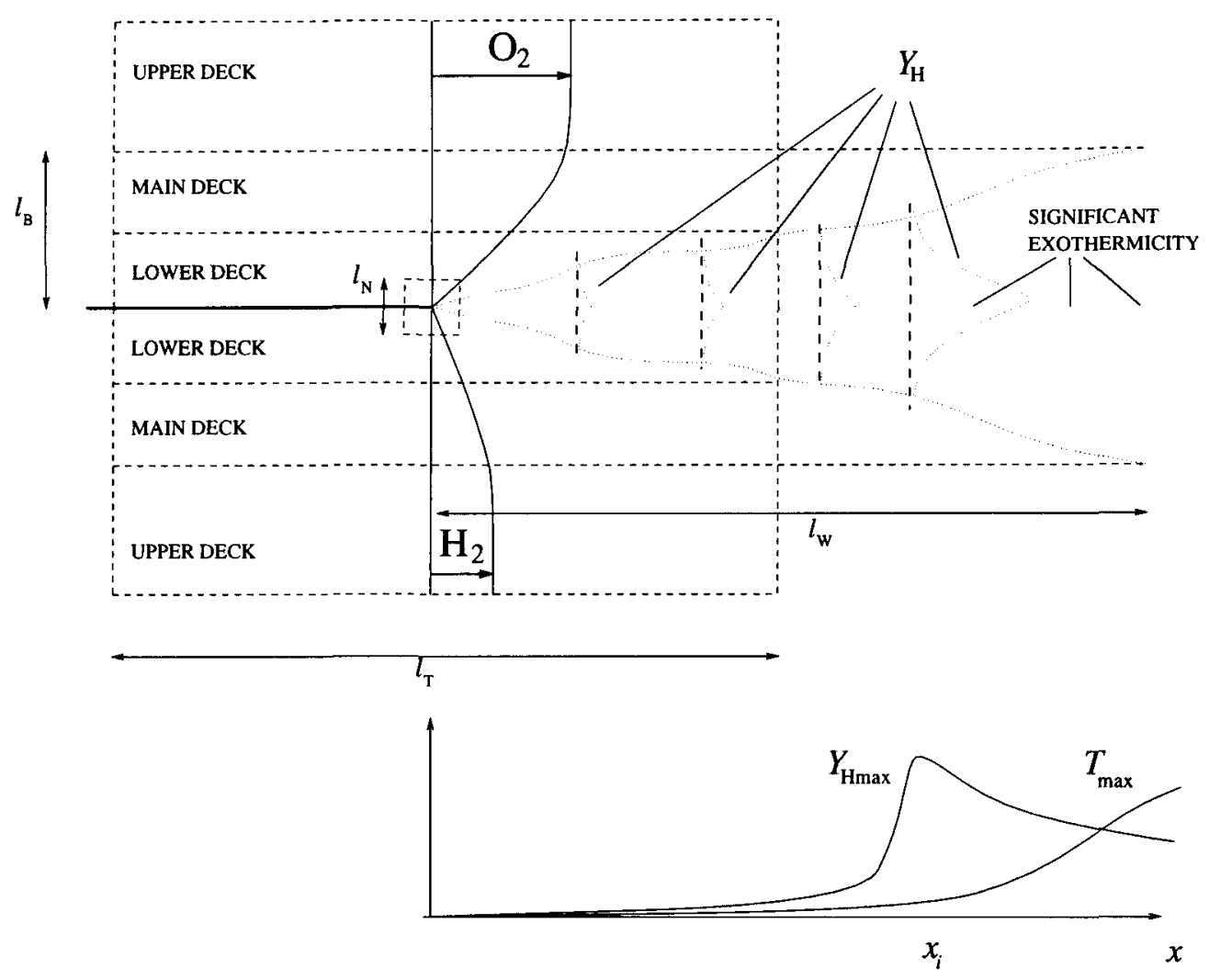

Figure 1. Triple-deck structure in the wake of a splitter plate.

The Reynolds number of the flow $R_{\mathrm{B}}=U l_{\mathrm{B}} / v$ based on the higher free-stream velocity $U$, boundary-layer thickness at the end of the splitter plate $l_{\mathrm{B}}$ and kinematic viscosity of the gas mixture in the wake $v$ is assumed to be large. Under those conditions, upstream conduction and diffusion can be neglected in the first approximation, except in a tiny region of characteristic size $l_{\mathrm{N}}=l_{\mathrm{B}} / R_{\mathrm{B}}^{1 / 2}$ around the edge of the plate, and the boundary-layer approximation can be adopted to describe the flow in the wake of the splitter plate [7], which exhibits the characteristic structure sketched in figure 1.

Balancing longitudinal convection and viscous terms in the streamwise momentum equation indicates that the characteristic length of the wake associated with the initial boundary layers $l_{\mathrm{W}}$ is a quantity of order $R_{\mathrm{B}} l_{\mathrm{B}}$, whereas its associated residence time is of order $t_{\mathrm{W}}=l_{\mathrm{B}}^{2} / \nu$ [7]. For values of the streamwise distance from the trailing edge, $x$, smaller than $l_{\mathrm{W}}$ the transverse extent of the mixing zone is small enough that matching the profile of streamwise velocity $u$ with the outer velocities involves only the slopes of the original boundary layers at the end of the splitter plate, $\lambda_{\infty}$ and $\lambda_{-\infty}$, with $\lambda_{\infty} \sim \lambda_{-\infty} \sim U / l_{\mathrm{B}}$. The resulting flow field consists of two outer regions of uniform vorticity separated by a viscous shear layer. The absence of characteristic length causes the solution in this region to be self-similar as pointed out by Goldstein [10]. At the upper edge of this region $u \simeq \lambda_{\infty} y$. Introducing this result into the streamwise momentum equation and equating again longitudinal convection and viscous terms indicates that the thickness of the mixing layer grows according to $\left(\nu x / \lambda_{\infty}\right)^{1 / 3}$, that the characteristic velocity is $\left(\lambda_{\infty}^{2} \nu x\right)^{1 / 3}$ and that 
the characteristic residence time is given by $t_{\mathrm{W}}\left(x / l_{\mathrm{W}}\right)^{2 / 3}$. Although the longitudinal pressure gradient $\mathrm{d} p / \mathrm{d} x$ is negligible in this Goldstein region, careful considerations $[13,14]$ reveal that a triple-deck region of characteristic length $l_{\mathrm{T}}=l_{\mathrm{B}} R_{\mathrm{B}}^{1 / 4}$ exists around the trailing edge of the plate, where the longitudinal pressure gradient self-induced by the evolution of the flow must be taken into account to describe the mixing process. It is seen that determination of $\mathrm{d} p / \mathrm{d} x$ requires consideration of the flow perturbations induced in the inviscid main and outer decks, resulting in a fairly complicated calculation [13, 14]. The solution is greatly simplified in the so-called Rott-Hakkinen region $[11,12]$, corresponding to distances $x \ll l_{\mathrm{T}}$ (but still much larger than $l_{\mathrm{N}}$ ), where the flow field also admits a self-similar description with the scales of the Goldstein region, but with a pressure gradient proportional to $x^{-1 / 3}$ that affects the mixing process at leading order.

In view of the above estimates of the ignition time $t_{i}=t_{\mathrm{B}} \ln \left(t_{\mathrm{I}} / t_{\mathrm{B}}\right)$ and of the residence time in the wake, $l_{\mathrm{B}}^{2} / \nu$, one can conclude that the previous chain-branching analyses $[4,5]$ apply only to configurations for which $l_{\mathrm{B}}^{2} / v<t_{\mathrm{B}} \ln \left(t_{\mathrm{I}} / t_{\mathrm{B}}\right)$. If this condition is not satisfied, then ignition occurs within the wake as sketched in figure 1 , and a new analysis is necessary. Although the problem can, in principle, be solved to determine the location of the ignition point also in the triple-deck region, for simplicity attention is restricted here to ignition events occurring in the Goldstein region $[10]\left(l_{\mathrm{T}} \ll x \ll l_{\mathrm{W}}\right)$ and in the Rott-Hakkinen region $[11,12]\left(l_{\mathrm{N}} \ll x \ll l_{\mathrm{T}}\right)$. The scalings given previously for the residence time indicate that the present analysis applies to configurations for which $R_{\mathrm{B}}^{-1} t_{\mathrm{W}} \ll t_{i} \ll R_{\mathrm{B}}^{-1 / 2} t_{\mathrm{W}}$ and $R_{\mathrm{B}}^{-1 / 2} t_{\mathrm{W}} \ll t_{i} \ll t_{\mathrm{W}}$. Clearly, ignition distances corresponding to configurations in the intermediate regimes $t_{i} \sim R_{\mathrm{B}}^{-1 / 2} t_{\mathrm{W}}$ and $t_{i} \sim t_{\mathrm{W}}$ can be quantified by appropriate extension of the present results and those previously obtained $[4,5]$.

\section{Formulation}

\subsection{Reduced kinetic mechanism}

If the temperature of the coflowing streams is sufficiently above crossover, the case considered in the present study, then the effect of three-body recombination reactions is negligible, and the initiation reaction

$$
\mathrm{H}_{2}+\mathrm{O}_{2} \stackrel{1}{\rightarrow} \mathrm{OH}+\mathrm{OH}
$$

and radical-branching reactions

$$
\begin{aligned}
& \mathrm{H}+\mathrm{O}_{2} \stackrel{2}{\rightarrow} \mathrm{OH}+\mathrm{O} \\
& \mathrm{H}_{2}+\mathrm{O} \stackrel{3}{\rightarrow} \mathrm{OH}+\mathrm{H}
\end{aligned}
$$

and

$$
\mathrm{H}_{2}+\mathrm{OH} \stackrel{4}{\rightarrow} \mathrm{H}_{2} \mathrm{O}+\mathrm{H}
$$

suffice to describe the branched-chain process of hydrogen-oxygen chemistry. In the ignition regime considered here the characteristic mass fractions of radicals and that of water vapour are small quantities, thereby causing the rates of the reverse reactions $2-4$, proportional to products of small mass fractions, to be negligible compared with the forward rates retained in the analysis, which are linearly proportional to radical mass fractions. The rates of the backward reactions $2-4$ only become significant very close to the ignition point, as the radical mole fractions increase to values of the order of unity, and they are consequently neglected in the analysis that follows. 
Radical growth downstream from the splitter plate is only significant in the wake, where low velocities exist and a longer residence time is available for chemical reactions to proceed. In this region, mixing provides sufficient molecular hydrogen to guarantee rapid consumption of $\mathrm{O}$ and $\mathrm{OH}$ through steps 3 and 4, thereby causing these two radicals to maintain steady state [8]. Introducing steady-state approximations for $\mathrm{O}$ and $\mathrm{OH}$ reduces the four-step mechanism to two identical overall reactions

$$
3 \mathrm{H}_{2}+\mathrm{O}_{2} \rightarrow 2 \mathrm{H}+2 \mathrm{H}_{2} \mathrm{O}
$$

with rates given by those of the elementary reactions 1 and 2 . The reaction-rate constants corresponding to steps $1-2$ are of the form $k_{j}=A_{j} T^{n_{j}} \exp \left[-E_{j} /\left(R^{o} T\right)\right]$, where $R^{o}$ is the universal gas constant. Updated values of the different reaction-rate parameters in $\mathrm{mol} \mathrm{cm}{ }^{-3}, \mathrm{~s}^{-1}, \mathrm{~K}$ and cal mol${ }^{-1}$ are $A_{1}=1.7 \times 10^{13}, A_{2}=3.52 \times 10^{16}, n_{1}=0$, $n_{2}=-0.7, E_{1}=47780$ and $E_{2}=17070$.

\subsection{Governing equations}

Since the chain-branching process is thermally frozen, upstream from the ignition point one can expect the temperature across the mixing layer to exhibit small deviations from the value of the temperature at the plate $T_{\mathrm{p}}$, and the density, $\rho$, and transport properties can be assumed to be constant in the wake if one neglects their mean-molecular weight variations. Because of the presence of hydrogen, retaining these variations would be necessary in undilute fuel-feed configurations for increased accuracy.

It is worth remarking that in typical applications of high-speed propulsion the oxidizer stream is supersonic. In that case, viscous dissipation upstream from the trailing edge of the splitter plate is a key factor in determining $T_{\mathrm{p}}$, causing relative temperature variations across the oxygen-side boundary layer of order $(\gamma-1) M_{\infty}^{2}$, where $\gamma$ is the ratio of specific heats and $M_{\infty}$ is the Mach number of the oxidizer stream. In the wake of the splitter plate, however, a straightforward order-of-magnitude analysis of the energy equation reveals that viscous dissipation only causes relative temperature increases from the splitter-plate temperature of order $(\gamma-1) M_{\infty}^{2}\left(x / l_{\mathrm{W}}\right)^{2 / 3}$, a quantity that remains small as long as ignition takes place within the wake of the splitter plate. Therefore, although viscous dissipation plays a fundamental role in analyses of ignition in supersonic boundary layers and mixing layers, its effect is limited to that of determining $T_{\mathrm{p}}$ in the present problem.

The pressure and velocity distributions, as well as the frozen reactant profiles, admit selfsimilar solutions that can be described by rescaling the transverse distance from the splitter plate $y$ with the characteristic local thickness of the wake to give the similarity coordinate $\eta=\left(\nu / \lambda_{\infty}\right)^{-1 / 3}\left(y / x^{1 / 3}\right)[10-12]$. Note that the value of $\lambda_{\infty}$ seen by the Rott-Hakkinen region is that at the trailing edge, whereas the value seen by the Goldstein region is that of the oncoming Blasius boundary layer upstream of the triple deck. Writing the streamwise velocity $u=\left(\nu \lambda_{\infty}^{2}\right)^{1 / 3} x^{1 / 3} F^{\prime}$ and transverse velocity $v=-\left(v^{2} \lambda_{\infty}\right)^{1 / 3} x^{-1 / 3}\left(2 / 3 F-1 / 3 \eta F^{\prime}\right)$ in terms of a nondimensional stream function $F(\eta)$, with the prime denoting differentiation with respect to $\eta$, reduces the momentum equation to

$$
F^{\prime \prime \prime}+\frac{2}{3} F F^{\prime \prime}-\frac{1}{3} F^{2}=C
$$

where $C$ is a similarity constant defined as $\mathrm{d} p / \mathrm{d} x=\rho\left(\nu \lambda_{\infty}^{2}\right)^{2 / 3} C x^{-1 / 3}$, to be obtained as part of the solution, and $C$ vanishes for the Goldstein region, where no pressure gradient exists. Integration of (1) with boundary conditions $F^{\prime}(\infty)=\eta$ and $F^{\prime}(-\infty)=\alpha \eta$ if $C \neq 0$ and $F^{\prime \prime}(\infty)=1$ and $F^{\prime \prime}(-\infty)=\alpha$ if $C=0$, where $\alpha=\lambda_{-\infty} / \lambda_{\infty}$ denotes the ratio of the shears at the bases of the two streams, can be performed by a simple shooting 
method as reported in the literature [11]. Note that the solution in the Goldstein region admits, in general, a vertical translation that is removed here by introducing the additional boundary condition $F(0)=0$. This translation is unimportant for the present analysis in that it does not modify the downstream location of the ignition point, its effect being only that of displacing transversely the resulting radical profiles.

Once the function $F$ is computed, one can integrate the similarity form of the frozen species conservation equations

$$
Y_{\mathrm{O}_{2} f}^{\prime \prime}+\frac{2}{3} S_{\mathrm{O}_{2}} F Y_{\mathrm{O}_{2} f}^{\prime}=Y_{\mathrm{H}_{2} f}^{\prime \prime}+\frac{2}{3} S_{\mathrm{H}_{2}} F Y_{\mathrm{H}_{2} f}^{\prime}=0
$$

with boundary conditions $Y_{\mathrm{O}_{2} f}-1=Y_{\mathrm{H}_{2} f}=0$ at $\eta=\infty$ and $Y_{\mathrm{O}_{2} f}=Y_{\mathrm{H}_{2} f}-1=0$ at $\eta=-\infty$ to determine the self-similar frozen reactant profiles

$$
Y_{\mathrm{O}_{2} f}=\frac{\int_{-\infty}^{\eta} \exp \left[-\frac{2}{3} S_{\mathrm{O}_{2}} \int_{0}^{\bar{\eta}} F(\tilde{\eta}) \mathrm{d} \tilde{\eta}\right] \mathrm{d} \bar{\eta}}{\int_{-\infty}^{\infty} \exp \left[-\frac{2}{3} S_{\mathrm{O}_{2}} \int_{0}^{\bar{\eta}} F(\tilde{\eta}) \mathrm{d} \tilde{\eta}\right] \mathrm{d} \bar{\eta}}
$$

and

$$
Y_{\mathrm{H}_{2} f}=1-\frac{\int_{-\infty}^{\eta} \exp \left[-\frac{2}{3} S_{\mathrm{H}_{2}} \int_{0}^{\bar{\eta}} F(\tilde{\eta}) \mathrm{d} \tilde{\eta}\right] \mathrm{d} \bar{\eta}}{\int_{-\infty}^{\infty} \exp \left[-\frac{2}{3} S_{\mathrm{H}_{2}} \int_{0}^{\bar{\eta}} F(\tilde{\eta}) \mathrm{d} \tilde{\eta}\right] \mathrm{d} \bar{\eta}} .
$$

Here, $Y_{\mathrm{O}_{2} f}$ and $Y_{\mathrm{H}_{2} f}$ represent the frozen mass fractions of the reactants scaled with their free stream values $Y_{\mathrm{O}_{2} \infty}$ and $Y_{\mathrm{H}_{2}-\infty}$, respectively, and $S_{i}$ denotes the Schmidt number of species $i$, with $D_{i}$ being its corresponding binary diffusion coefficient. Reactant distributions obtained from the above quadratures are exhibited, for instance, in [6].

As shown below, the radical pool grows very rapidly prior to ignition. Consequently, the influence of reactant consumption upstream from the ignition point can be neglected in the first approximation, thereby reducing the problem to that of integrating the $\mathrm{H}$-atom conservation equation with the chemical terms calculated with frozen reactant concentrations and with the reaction-rate constants evaluated at $T_{\mathrm{p}}$. To write this equation, it is convenient to employ the similarity coordinate $\eta$ together with a normalized streamwise coordinate $\xi=\left(2 \rho Y_{\mathrm{O}_{2} \infty} k_{2} / W_{\mathrm{O}_{2}}\right)^{3 / 2} x /\left(\nu^{1 / 2} \lambda_{\infty}\right)$ and a normalized $H$-atom mass fraction $y_{\mathrm{H}}=\left(2 k_{2} / k_{1}\right)\left(Y_{\mathrm{H}} / Y_{\mathrm{H}_{2}-\infty}\right)$ to give in the first approximation

$$
\xi^{1 / 3} F^{\prime} \frac{\partial y_{\mathbf{H}}}{\partial \xi}-\frac{1}{\xi^{2 / 3}}\left[\frac{2}{3} F y_{\mathrm{H}}^{\prime}+\frac{1}{S_{\mathrm{H}}} y_{\mathrm{H}}^{\prime \prime}\right]=Y_{\mathrm{O}_{2} f}\left(Y_{\mathrm{H}_{2} f}+y_{\mathrm{H}}\right)
$$

with boundary conditions $y_{\mathrm{H}}=0$ at $\xi=0$ and also as $\eta \rightarrow \pm \infty$ for $\xi>0$.

\section{Radical growth in the wake}

To help us expose the different regions appearing in the igniting wake, the ratio of the characteristic time of radical branching to the characteristic time of initiation evaluated at $T_{\mathrm{p}}, \varepsilon=k_{1} /\left(2 k_{2}\right)$, has been used as a scale for the $\mathrm{H}$-atom mass fraction. This quantity, typically very small with values at $T_{\mathrm{p}}=1200$ and $T_{\mathrm{p}}=2000$ given approximately by $8.36 \times 10^{-8}$ and $2.1 \times 10^{-5}$, is a measure of the characteristic radical mass fraction for which the rates of the initiation and branching steps are equal.

Observation of (5) reveals that for values of $y_{\mathrm{H}} \ll 1$, corresponding to $\xi \ll 1$, radical growth is dominated by the initiation reaction, giving radical mass fractions initially increasing with downstream distance according to $y_{H} \propto \xi^{2 / 3}$. This initiation-controlled region ends as $y_{\mathrm{H}}$ reaches values of order unity, corresponding to $\mathrm{H}$-atom mass fractions of order $\varepsilon$, for which the branching and initiation steps are approximately equal. In the following intermediate region both $y_{\mathrm{H}}$ and $\xi$ are of order unity, and all transport and 
chemical terms in (5) are equally important. As $y_{\mathrm{H}}$ further increases to values larger than unity, the initiation term becomes negligible as can be seen from (5), ushering in a region of radical growth controlled by the branching step. Since the values of the radical mass fractions corresponding to partial equilibrium of the branching reactions are typically of order unity, i.e. the value of $y_{H}$ at ignition is a large quantity of characteristic value $\varepsilon^{-1}$, this third autocatalytic stage extends over most of the ignition process, taking place over a long distance corresponding to $\xi$ in the range $1 \ll \xi \ll\left[\ln \left(\varepsilon^{-1}\right)\right]^{3 / 2}$ as shown below. As a consequence, if errors of order unity (small relative errors of order $\left[\ln \left(\varepsilon^{-1}\right)\right]^{-3 / 2}$ ) are neglected, then one can calculate the ignition distance $\xi_{i}$ by investigating the solution in the autocatalytic growth region, where the coordinate $\xi \gg 1$ can be used as an asymptotically large quantity to simplify the solution.

\subsection{The asymptotic expansion}

In the autocatalytic growth region $y_{\mathrm{H}} \gg 1$ and the initiation term can be neglected in (5), yielding

$$
\frac{\partial \psi}{\partial \tau}-\frac{1}{\tau F^{\prime}(\eta)}\left[F(\eta) \psi^{\prime}+\frac{3}{2 S_{\mathrm{H}}}\left(\psi^{\prime \prime}+\psi^{\prime 2}\right)\right]=h(\eta)
$$

where $\psi=\ln \left(y_{\mathrm{H}}\right)$ is a new variable that helps to expose the exponential nature of the radical growth behaviour and $\tau=\xi^{2 / 3}$ is the ratio of the characteristic residence time in the wake $x^{2 / 3} /\left(v^{1 / 3} \lambda_{\infty}^{2 / 3}\right)$ to the characteristic branching time $\left(2 \rho Y_{\mathrm{O}_{2} \infty} k_{2} / W_{\mathrm{O}_{2}}\right)^{-1}$. The boundary conditions $y_{\mathrm{H}} \rightarrow 0$ as $\eta \rightarrow \pm \infty$ transform to $\psi( \pm \infty)=-\infty$. The function $h=\frac{3}{2} Y_{\mathrm{O}_{2} f}(\eta) / F^{\prime}(\eta)$ is a reduced Damköhler number defined with the local flow velocity and local oxidizer mass fraction. For configurations with nonzero values of the parameter $\alpha$, this function vanishes as $\eta \rightarrow \pm \infty$, where the residence time decreases to zero, exhibiting a maximum value $h\left(\eta^{*}\right)$ at an intermediate transverse location $\eta^{*}$.

Solutions to (6) in the limit $\tau \gg 1(\xi \gg 1)$ can be obtained by introducing an expansion for $\psi$ of the form

$\psi=\tau G_{0}(\eta)+\tau^{1 / 2} G_{1}(\eta)+\frac{3}{2} \ln (\tau) G_{2}(\eta)+G_{3}(\eta)+\tau^{-1 / 2} G_{4}(\eta)+\cdots$

with $G_{0}, G_{1}, \ldots$ being functions of order unity and the factor $\frac{3}{2}$ in the third term being included for convenience in the presentation of the results. It will be seen below that the terms of orders $\tau$ and $\ln (\tau)$, which do not emerge from dominant balance considerations in the asymptotic analysis, are switchback terms that must be included in the expansion to provide a bounded, nonoscillatory $\mathrm{H}$-atom profile. Introducing (7) into (6) and collecting terms of the same order in powers of $\tau$ enables the problem to be solved sequentially as follows.

\subsection{Leading-order uniform growth}

At the lowest order $(\tau)$ the single-term equation

$$
G_{0}^{\prime 2}=0
$$

indicates that, because of radical diffusion, radical growth is uniform across the mixing layer in the first approximation. Since a constant value of $G_{0}=G_{0}^{*}$ satisfies identically the equation of order $\tau^{1 / 2}$,

$$
G_{0}^{\prime} G_{1}^{\prime}=0
$$


investigation of the equation that appears at order unity

$$
G_{1}^{\prime 2}=\frac{2}{3} S_{\mathrm{H}} F^{\prime}\left(G_{0}^{*}-h\right)
$$

is necessary for determining the value of $G_{0}^{*}$. Solving (10) for $G_{1}^{\prime}$ yields

$$
G_{1}^{\prime}= \pm\left(\frac{2}{3} S_{\mathrm{H}} F^{\prime}\right)^{1 / 2}\left(G_{0}^{*}-h\right)^{1 / 2}
$$

a function of $\eta$ that exhibits a different number of zeros depending upon the value of $G_{0}^{*}$. Thus, for values of $G_{0}^{*}$ larger than the maximum reduced Damköhler number, $h\left(\eta^{*}\right)$, the function $G_{1}^{\prime}$ never vanishes, thereby ca'ssing the solution for $G_{1}$ associated with the plus (respectively minus) sign to increase (decrease) monotonically with $\eta$. Clearly, the resulting $\psi$ profiles cannot satisfy simultaneously the two boundary conditions $\psi( \pm \infty)=-\infty$ and, consequently, values of $G_{0}^{*}$ larger than $h\left(\eta^{*}\right)$ must be disregarded. Similarly, for values of $G_{0}^{*}$ below $h\left(\eta^{*}\right)$ there exist two zeros of $G_{1}^{\prime}$, between which the resulting value of $G_{1}^{\prime}$ would be imaginary. This solution corresponds to oscillatory radical profiles, a behaviour that is not physically acceptable. Therefore, the asymptotic solution must present a single zero for $G_{1}^{\prime}$, a condition that yields the equation

$$
G_{0}^{*}=h\left(\eta^{*}\right)
$$

indicating that the leading-order exponential growth corresponds to that of the transverse location $\eta^{*}$ where the local Damköhler number is maximum. Note that, although transverse radical diffusion causes the leading-order growth to be uniform everywhere across the mixing layer, the resulting leading-order rate of radical branching is independent of radical diffusivity.

The results also indicate that the $\mathrm{H}$-atom profile peaks at $\eta^{*}$, decreasing towards the free streams as described by integrating (11) to give

$$
G_{1}=G_{1}^{*} \pm\left(\frac{2}{3} S_{\mathrm{H}}\right)^{1 / 2} \int_{\eta^{*}}^{\eta}\left(F^{\prime}\right)^{1 / 2}\left(G_{0}^{*}-h\right)^{1 / 2} \mathrm{~d} \eta
$$

where the plus and minus signs correspond, respectively, to $\eta<\eta^{*}$ and to $\eta>\eta^{*}$. The constant $G_{1}^{*}=G_{1}\left(\eta^{*}\right)$ in (13) remains undetermined in the integration, its value being obtained below as part of the analysis at the following order by imposing a condition of bounded behaviour on the solution at $\eta=\eta^{*}$.

\subsection{Radical-diffusion correction}

Carrying on the analysis to the following order $\left(\tau^{-1 / 2} \ln (\tau)\right)$ yields $G_{2}^{\prime}=0$, indicating that the logarithmic term in (7) reduces to a uniform contribution $G_{2}=G_{2}^{*}$ that needs to be included to guarantee the boundness of $G_{4}^{\prime}$ as shown below. The next nontrivial equation, found at order $\tau^{-1 / 2}$, can be solved for $G_{3}^{\prime}$ to give

$$
G_{3}^{\prime}=S_{\mathrm{H}}\left[\frac{1}{2}\left(F^{\prime} G_{1} / 3-G_{1}^{\prime \prime} / S_{\mathrm{H}}\right) / G_{1}^{\prime}-\frac{1}{3} F\right] .
$$

The expression in parentheses on the right-hand side of the above equation must vanish at $\eta=\eta^{*}$ (where $G_{1}^{\prime}=0$ ) to avoid a singular behaviour, a condition that determines the value of $G_{1}^{*}$. Expanding (11) in a Taylor series around $\eta=\eta^{*}$ to evaluate $G_{1}^{\prime \prime}$ gives

$$
G_{1}^{*}=-\left(\frac{3}{S_{\mathrm{H}} F^{\prime}}\right)^{1 / 2}\left(-h^{\prime \prime}\right)^{1 / 2}
$$

as an expression for the second-order correction to the growth rate at $\eta=\eta^{*}$. The local velocity $F^{\prime}$ and the curvature of the Damköhler-number distribution $h^{\prime \prime}$ appearing in (15) must be evaluated at $\eta=\eta^{*}$. This term gives a negative contribution that accounts for 
radical diffusion from the radical peak. As expected [5], radical migration towards the free streams becomes more significant for larger values of the radical diffusivity $\left(G_{1}^{*} \propto S_{\mathrm{H}}^{-1 / 2}\right.$ ), and also as the radical profile becomes sharper at its peak $\left(G_{1}^{*} \propto\left(-h^{\prime \prime}\right)^{-1 / 2}\right)$.

Before proceeding with the computation of $G_{2}^{*}$, it is worth remarking that limitations to the present asymptotic analysis appear already at order unity, causing the constant emerging in the integration of (14), $G_{3}^{*}$, to remain necessarily undetermined. This can be easily proven by noticing that a solution to (6), the starting equation obtained by removing the initiation term, admits an arbitrary additive constant, indicating that the value of $G_{3}^{*}$ mentioned above carries the memory effects associated with the initial region where the initiation reaction is significant. Consequently, $G_{3}^{*}$ can only be obtained by matching the present asymptotic solution with the profile emerging as $\tau \gg 1$ from numerical integration of (5). Although the associated matching process is not pursued further here, we shall show below that the first three terms in (7) provide sufficient accuracy for moderately large values of $\tau$.

\subsection{The intermediate logarithmic term}

Although two-term expansions were employed in previous mixing-layer chain-branching analyses [4, 5], it will be shown below that the logarithmic term in (7) gives in this case a non-negligible contribution for values of $\tau$ of practical interest. To obtain the value of $G_{2}^{*}$, one needs to investigate the equation that appears at order $\tau^{-1}$

$$
G_{2}^{*}-\frac{2}{3} \frac{F}{F^{\prime}} G_{3}^{\prime}-\frac{1}{S_{\mathrm{H}} F^{\prime}} G_{3}^{\prime \prime}-\frac{1}{S_{\mathrm{H}} F^{\prime}}\left(2 G_{1}^{\prime} G_{4}^{\prime}+G_{3}^{\prime 2}\right)=0 .
$$

Following the previous reasoning used in the calculation of $G_{1}^{*}$, one may easily conclude that the boundness of the solution at $\eta=\eta^{*}$ requires that

$$
G_{2}^{*}=\frac{1}{S_{\mathrm{H}} F^{\prime}}\left(\frac{2}{3} S_{\mathrm{H}} F G_{3}^{\prime}+G_{3}^{\prime 2}+G_{3}^{\prime \prime}\right)
$$

where the functions $F, F^{\prime}$,

$$
G_{3}^{\prime}=-\frac{1}{3}\left(S_{\mathrm{H}} F+\frac{1}{2} \frac{h^{\prime \prime \prime}}{h^{\prime \prime}}\right)
$$

and

$$
G_{3}^{\prime \prime}=-\frac{1}{4}\left(\frac{1}{4} \frac{h^{\prime \prime \prime \prime}}{h^{\prime \prime}}-\frac{7}{36} \frac{h^{\prime \prime \prime 2}}{h^{\prime 2}}-\frac{3}{4} \frac{F^{\prime 2}}{F^{\prime 2}}+\frac{1}{6} \frac{F^{\prime \prime}}{F^{\prime}} \frac{h^{\prime \prime \prime}}{h^{\prime \prime}}+S_{\mathrm{H}} F^{\prime}+\frac{1}{2} \frac{F^{\prime \prime \prime}}{F^{\prime}}\right)
$$

must be evaluated at $\eta=\eta^{*}$, with the expressions for $G_{3}^{\prime}$ and $G_{3}^{\prime \prime}$ obtained from Taylor expansions of (11) and (14) around $\eta=\eta^{*}$.

\section{Discussion of the asymptotic results}

The order of the approximation adopted here yields

$$
\begin{aligned}
y_{\mathbf{H}}=\exp \left[\xi^{2 / 3} G_{0}^{*}+\xi^{1 / 3} G_{1}^{*}+\ln (\xi) G_{2}^{*}\right] \\
\times \exp \left[ \pm \xi^{1 / 3}\left(\frac{2}{3} S_{\mathrm{H}}\right)^{1 / 2} \int_{\eta^{*}}^{\eta}\left(F^{\prime}\right)^{1 / 2}\left(G_{0}^{*}-h\right)^{1 / 2} \mathrm{~d} \eta\right]
\end{aligned}
$$

as a representation for the radical profiles, where the first exponential gives the variation of the maximum radical mass fraction with distance, while the second exponential reproduces the shape of the radical profile at leading order. Values of $G_{0}^{*}, G_{1}^{*}$ and $G_{2}^{*}$ obtained from (12), (15) and (17), as well as the corresponding value of $\eta^{*}$ at which the Damköhler 


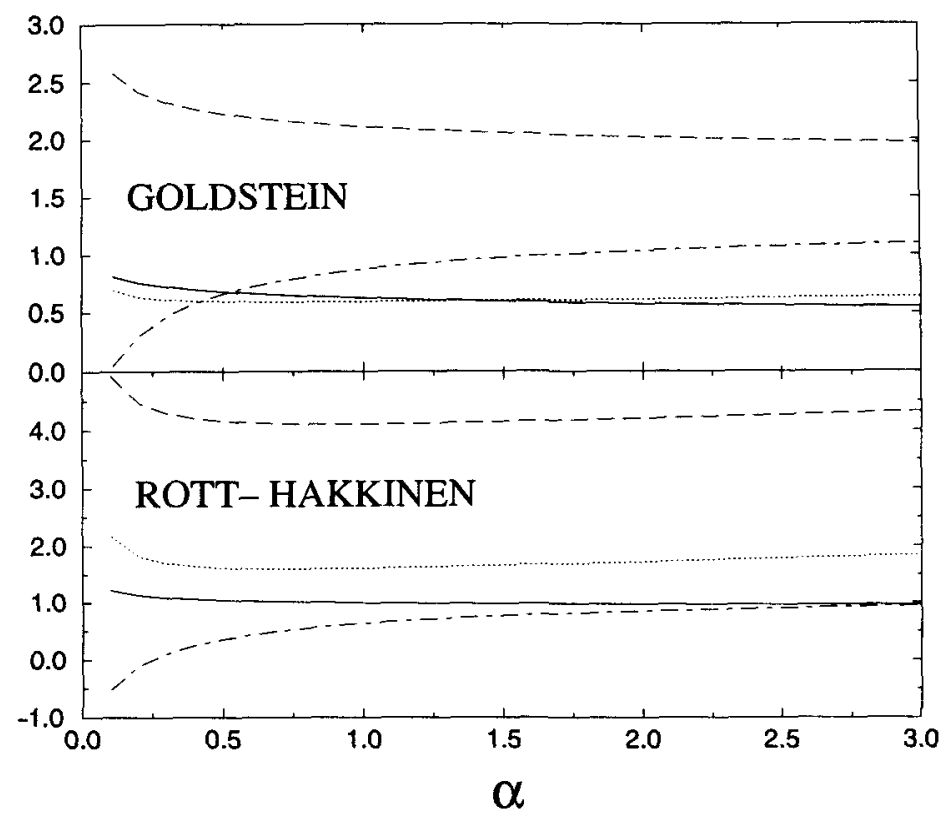

Figure 2. The variation with $\alpha$ of $G_{0}^{*}$ (full curve), $-G_{1}^{*}$ (broken curve) and $G_{2}^{*}$ (dotted curve) as obtained from (12), (15) and (17), respectively, together with the corresponding value of the transverse location $\eta^{*}$ where the function $h(\eta)$ peaks (chain curve).

number peaks, are plotted in figure 2 as a function of $\alpha$ for both the Goldstein and the Rott-Hakkinen regions, and with the values $S_{\mathrm{O}_{2}}=0.74, S_{\mathrm{H}_{2}}=0.19$ and $S_{\mathrm{H}}=0.12$ adopted in the calculations.

The first-order $\exp \left[\xi^{2 / 3} G_{0}^{*}\right]$, second-order $\exp \left[\xi^{2 / 3} G_{0}^{*}+\xi^{1 / 3} G_{1}^{*}\right]$ and third-order $\exp \left[\xi^{2 / 3} G_{0}^{*}+\xi^{1 / 3} G_{1}^{*}+\ln (\xi) G_{2}^{*}\right]$ asymptotic predictions for the maximum H-atom mass fraction are compared with results of numerical integrations of (5) in figure 3 . The numerical integrations were performed by means of a Crank-Nicolson procedure with an iterative scheme adopted for the solution of the implicit nonlinear system of equations in $\eta$ that appear at each $\xi$. To handle the unbounded value of $\partial y_{H} / \partial \xi$ at $\xi=0$ the initial condition $y_{H}=0$ at $\xi=0$ was replaced in the numerical integrations by the leading-order representation of the radical mass fraction for small values of $\xi, y_{\mathrm{H}}=\xi^{2 / 3} \phi_{0}$, evaluated at $\xi \ll 1$. The function $\phi_{0}=\phi_{0}(\eta)$ is determined as the solution to the ordinary differential equation

$$
\frac{1}{S_{\mathrm{H}}} \phi_{0}^{\prime \prime}+\frac{2}{3} F \phi_{0}^{\prime}-\frac{2}{3} F^{\prime} \phi_{0}=-Y_{\mathrm{O}_{2} f} Y_{\mathrm{H}_{2} f}
$$

with boundary conditions $\phi_{0}( \pm \infty)=0$, obtained by substituting the asymptotic expansion $y_{\mathrm{H}}=\xi^{2 / 3} \phi_{0}+\xi^{4 / 3} \phi_{1}+\cdots$, valid for $\xi \ll 1$, into (5) and collecting the leading terms in powers of $\xi$.

The comparisons, which correspond to the Rott-Hakkinen mixing layer with $\alpha=1$, clearly show that a three-term expansion is necessary to provide sufficient accuracy for ignition distances. Similar behaviours are found in the Goldstein region and also for values of $\alpha$ different from unity, further suggesting that, while a two-term expansion suffices to describe ignition histories in the mixing layer outside of the wake $[4,5]$, the contribution of the logarithmic correction remains significant up to the ignition point when ignition takes place in the wake. Solving $\exp \left[\xi^{2 / 3} G_{0}^{*}+\xi^{1 / 3} G_{1}^{*}+\ln (\xi) G_{2}^{*}\right]=\varepsilon^{-1}=2 k_{2} / k_{1}$ gives with 


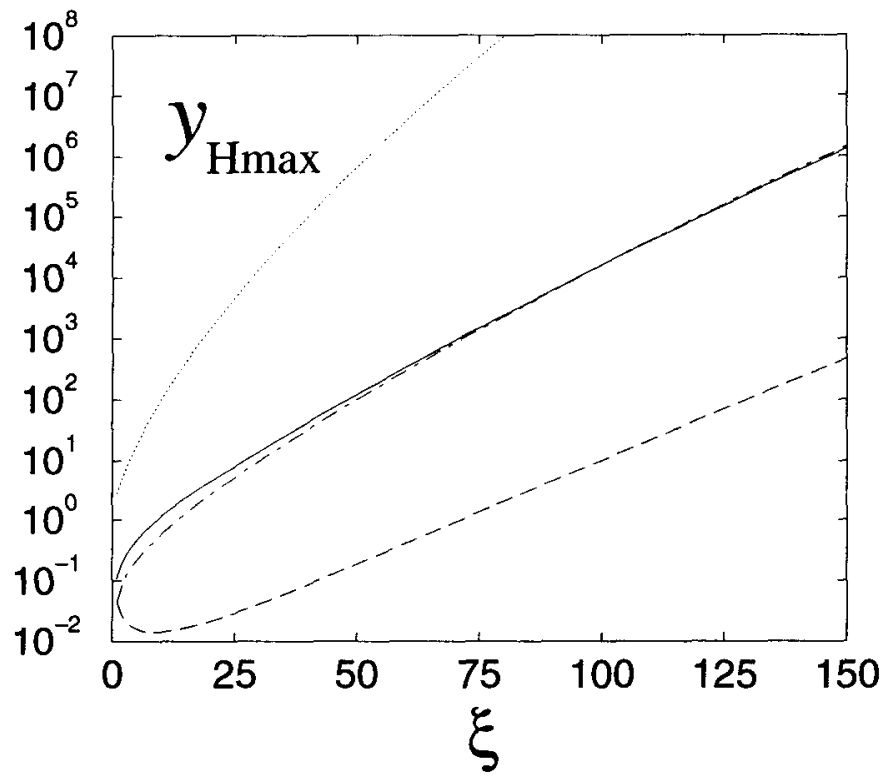

Figure 3. The evolution with downstream distance of the maximum value of $y_{H}$ (full curve) obtained by integration of (5) for the Rott-Hakkinen mixing layer with $\alpha=1$, and comparison with the leading-order $\exp \left[\xi^{2 / 3} G_{0}^{*}\right]$ (dotted curve), second-order $\exp \left[\xi^{2 / 3} G_{0}^{*}+\xi^{1 / 3} G_{1}^{*}\right]$ (broken curve) and third-order $\exp \left[\xi^{2 / 3} G_{0}^{*}+\xi^{1 / 3} G_{1}^{*}+\ln (\xi) G_{2}^{*}\right]$ (chain curve) asymptotic predictions.

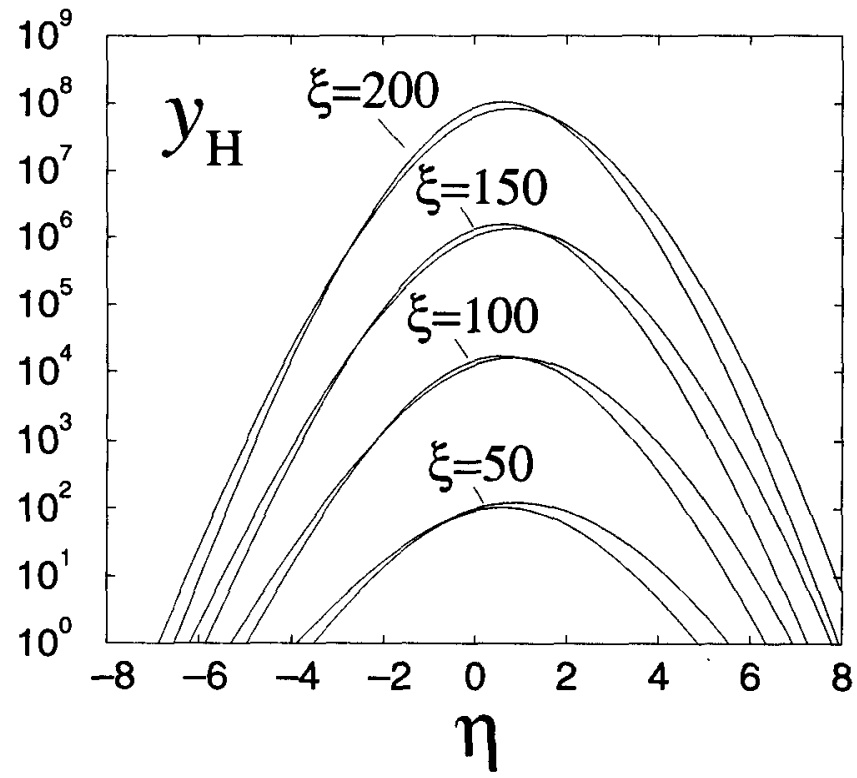

Figure 4. A comparison for $\xi=50, \xi=100, \xi=150$ and $\xi=200$ of the $\mathrm{H}$-atom profile corresponding to the Rott-Hakkinen mixing layer with $\alpha=1$ obtained from integration of (5) (full curve) with the asymptotic prediction given in (20) (broken curve). 
relative errors of order $\left[\ln \left(\varepsilon^{-1}\right)\right]^{-3 / 2}$

$$
\xi_{i}=\left[\frac{\ln \left(\varepsilon^{-1}\right)}{G_{0}^{*}}\right]^{3 / 2}\left[1-\frac{1}{\ln \left(\varepsilon^{-1}\right)^{1 / 2}} \frac{3}{2} \frac{G_{1}^{*}}{\left(G_{0}^{*}\right)^{1 / 2}}-\frac{\ln \left[\ln \left(\varepsilon^{-1}\right)\right]}{\ln \left(\varepsilon^{-1}\right)} \frac{9}{4} G_{2}^{*}\right]
$$

as an explicit expression for the ignition distance.

The shape of the $\mathrm{H}$-atom profiles appears in the asymptotic expansion at order $\xi^{1 / 3}$ as seen in the second exponential factor of $(20)$, thereby enabling the computation of radical profiles with errors of order unity. To test the accuracy of this prediction, the profiles given by (20) are compared in figure 4 at different downstream locations with those emerging from numerical integrations of (5) for the Rott-Hakkinen mixing layer with $\alpha=1$. As can be seen, the asymptotic prediction is excellent everywhere, describing with errors of order unity the large transverse variation of radical mass fraction across the mixing layer. Somewhat larger errors are found as the oxidizer boundary is approached. An explanation for these departures can be readily found by observing from (11) that as $\eta \rightarrow \pm \infty$ the absolute value of $G_{1}$ increases according to $G_{1} \propto \eta^{3 / 2}$. Comparing then the first two terms in (7) reveals that the asymptotic analysis presented here breaks down at transverse distances $\eta$ of order $\tau^{1 / 3}=\xi^{2 / 9}$. Therefore, although the range of validity of (20) increases with increasing $\xi$, departures from the asymptotic predictions can be expected for $\left|\eta-\eta^{*}\right|>\xi^{2 / 9}$.

\section{Conclusions}

We have extended here previous analyses of chain-branching explosions in $\mathrm{H}_{2}-\mathrm{O}_{2}$ mixing layers $[4,5]$ by taking into account the effect of the wake that forms at the trailing edge of the splitter plate initially separating the reactants. An asymptotic description for the $\mathrm{H}$-atom profile is found in terms of a WKB-like exponential expansion that employs as a large asymptotic parameter $\xi$, the ratio of the streamwise distance from the splitter plate to the downstream extent of the initial region where the initiation reactions are significant. It is seen that three terms, including a switchback logarithmic term not calculated in previous analyses [4, 5], must be retained for an accurate description of radical growth. An important result of the analysis is the prediction of $\xi_{i}$ given in (22), which, together with the parametric results presented in figure 2 , enable the accurate computation of ignition distances within the wake.

A related problem not addressed here is that of the effect of the splitter-plate thickness on the ignition distance. In general, at the trailing edge of a splitter plate of finite thickness $H$ there exists a recirculating region of characteristic residence time $H^{2} / v$. In view of previous results [15-17], it can be anticipated that the solution that appears in the near wake of the splitter plate depends on the relative value of the residence time and branching time of the underlying chemistry. Thus, if the plate thickness is sufficiently small, then a quasifrozen solution with characteristic radical mass fractions of the order of the ratio of the characteristic branching time to the characteristic initiation time exists in the vicinity of the trailing edge. In this case, chain growth proceeds slowly downstream as described in the present paper, giving rise to radical concentrations continuously increasing with distance, and to flame development downstream at distances from the splitter plate large compared to the size of the recirculating region. This quasifrozen near-wake solution ceases to exist when the residence time $H^{2} / v$ reaches a critical value of the order of the branching time, ushering in a regime in which relatively large radical mass fractions are already present in the vicinity of the trailing edge, with the criticality of the resultant bifurcation being governed by the competition of heat release and reactant consumption. In that case, the ignition point at which the branching reactions reach partial equilibrium is reached a short 
distance downstream from the recirculating region, an important result in connection with high-speed propulsion applications.

The work of ALS was supported by the Spanish DGICYT under contract no PB95-0296, while that of II was supported by the Spanish DGICYT under contract no PB95-0280 and that of AL was supported by the Spanish CICYT under contract no PB94-0400 and by INTA under contract no INTA 4070-0036/1995.

Liñán A and Crespo A 1976 Combust. Sci. Tech. 1495

Ju Y and Niioka T 1994 Combust. Flame 99240

Treviño C and Liñán A 1995 Combust. Flame 103129

Sánchez A L, Liñán A and Williams F A 1997 J. Eng. Math. 31119

Sánchez A L, Liñán A and Williams F A 1998 SIAM J. Appl. Math. to be published

Sánchez A L 1997 Phys. Fluids 92032

Liñán A 1994 Combustion in High Speed Flows ed J Buckmaster, T L Jackson and A Kumar (New York: Kluwer) pp 461-76

Treviño C 1991 Progress in Astronautics and Aeronautics, AIAA 13119

Sánchez A L, Liñán A and Williams F A 1997 Combust. Sci. Tech. 123317

Goldstein S 1930 Proc. Camb. Philos. Soc. 261

Rott N and Hakkinen R J 1962 J. Aerospace Sci. 291134

Hakkinen R J and Rott N 1965 AIAA J. 31553

Stewartson K 1969 Mathematika 16106

Messiter A F 1970 SIAM J. Appl. Math. 18241

Darabiha N and Candel S 1992 Combust. Sci. Tech. 8667

Sánchez A L, Liñán A and Williams F A 1994 Proc. 25th Int. Symp. on Combustion (Pittsburgh, PA: Combustion Institute) p 1529

Sánchez A L, Balakrishnan G, Liñán A and Williams F A 1996 Combust. Flame 105569 